\title{
Design and Implementation of the Remote Wireless Intraoral Endoscope System
}

\author{
Zhangong Xie ${ }^{1,2, a}$, Xiao Zhang ${ }^{1,2, b}$, Dewen Zeng $^{\star, 3, c}$, Xianshuai Chen ${ }^{1,4, d}$, Wei \\ Feng $2,4, e$ \\ ${ }^{1}$ Center of Precision Engineering, Guangzhou Institute of Advanced Technology, Chinese Academy \\ of Sciences (GIAT), Guangzhou, 511458, China \\ ${ }^{2}$ Shenzhen Institute of Advanced Technology, Chinese Academy of Sciences(SIAT), Guangzhou, \\ 518055, China \\ ${ }^{3}$ Center of Intelligent Control, Guangzhou Institute of Advanced Technology, Chinese Academy of \\ Sciences (GIAT), Guangzhou, 511458, China \\ ${ }^{4}$ The Chinese University of Hong Kong \\ *Dewen Zeng is the corresponding author

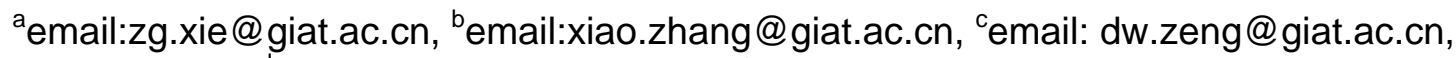 \\ demail: xs.chen@giat.ac.cn, e email: wei.feng@siat.ac.cn
}

Keywords:Oral health; Intraoral Endoscope; remote; wireless

\begin{abstract}
Oral health is one of the most important parts of the body health. It is very significant to periodically inspect the status of our oral cavity so as to maintain and improve the quality of our lives. Recently, oral inspection is usually carried out only in the hospital. People are unable to inspect their oral cavity by their own due to lack of professional tools and specialized knowledge. Therefore, a Remote Wireless Intraoral Endoscope System is proposed in this paper, which is consisted of two parts: a probe for wirelessly collecting the image of oral cavity by using a CMOS imaging sensor and an interactive display unit for both displaying the image collected by the probe and interacting with remote server. Our design and implementation shows the validity and practicability of the system.
\end{abstract}

\section{Introduction}

Oral health is one of the most important parts of the body health [1]. Common oral disease such as dental caries and periodontal diseases are mostly chronic diseases. The incipient symptoms of these diseases are relatively not obvious, which are prone to be neglected by people [2][3]. Intraoral endoscopes are the indispensable tools for dentists [4], which is composed of a camera system and a display system, through which the photos of the intraoral condition can be collected and transmitted to the monitors. Currently, most of intraoral endoscopes must be cooperated with the medical equipment which is mainly possessed by hospitals or outpatient services. This means people must directly go to these places to carry out intraoral inspection if they want to know about their oral condition. Therefore, only a few of people periodically go to the hospital to inspect their oral cavity, which is detrimental to the normal treatment and people's own health.

Motivated by the challenges mentioned above, a Remote Wireless Intraoral Endoscope System is designed in this paper to provide hardware and software supports of the remote inspection system and finally implement the intraoral inspection whenever and wherever possible conveniently [5-7]. This system is able to not only collect the images of the oral cavity, but also to transmit these images data to the data center wirelessly. By analyzing the relevant data, specialist can communicate with patients online so that people can know their intraoral condition at home instead of coming to the hospital.

The rest of this paper is organized as follows. The operation principle of our Intraoral Endoscope system is explained in section II. The hardware and software design is presented in section III and section IV, respectively. The results and analysis of our proposed system is shown in section V. 
Finally, section VI concludes this paper.

\section{Operation Principle}

A complete Wireless Remote Intraoral Inspection System consists of an inspection terminal for users and a back-end server for doctors. The offline and online operation modes of these two terminals are shown in Fig.1 and Fig.2, respectively. Users can not only collect the images of their oral cavity to initially observe the intraoral condition, but also can communicate with specialists or doctors online to acquire professional advices and therapeutic plans.
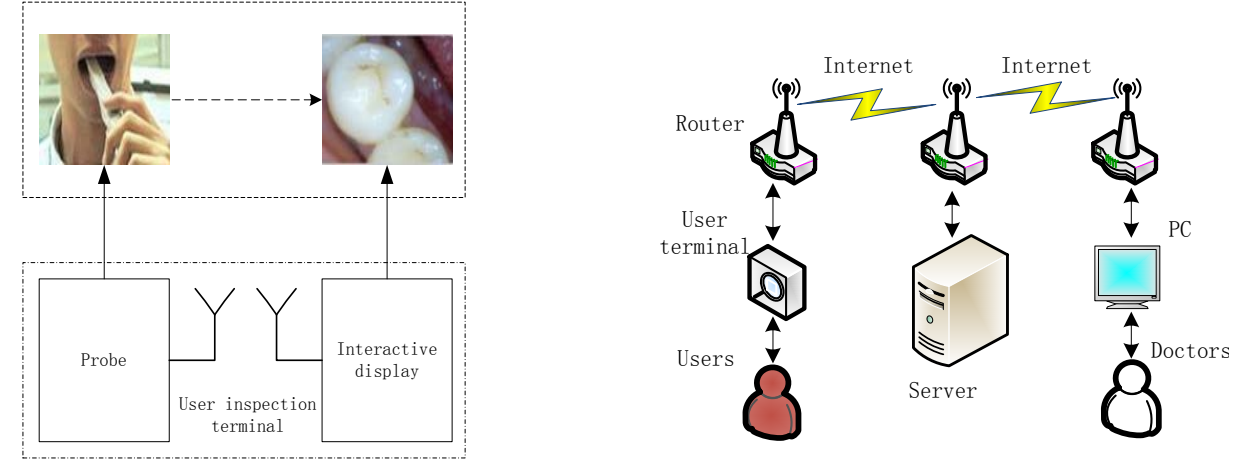

Fig.1. Offline mode for intraoral endoscope. Fig.2.Online mode for intraoral endoscope.

Under offline mode, users can observe their oral cavity on the screen of interactive display through simply put the probe unit into their mouth and take photos of interest [8] [9], while under online mode, images are sent to the remote server, which can be analyzed in real-time by doctors who will then offer professional advices back to the users [10] [11]. No matter which mode is being used, the inspection terminal is an essential part, which is consisted of a probe unit and an interactive display unit. In our design, these two units are connected wirelessly which will be presented in detail in the following sections.

A. The function and principle of the probe unit.

The probe is used for collecting the images of the oral cavity and transmitting them to the interactive display for observation. Because the light is insufficient in the oral environment, an extra illuminant must be equipped to provide enough illumination. Moreover, probe with cables will cause inconvenience when taking photos of oral cavity, hence, connect probe and interactive display without cables should be considered as well. In our design, batteries are utilized for power supply and wireless communication technology is chosen for data transmission. The block diagram of the probe unit is shown in Fig.3.
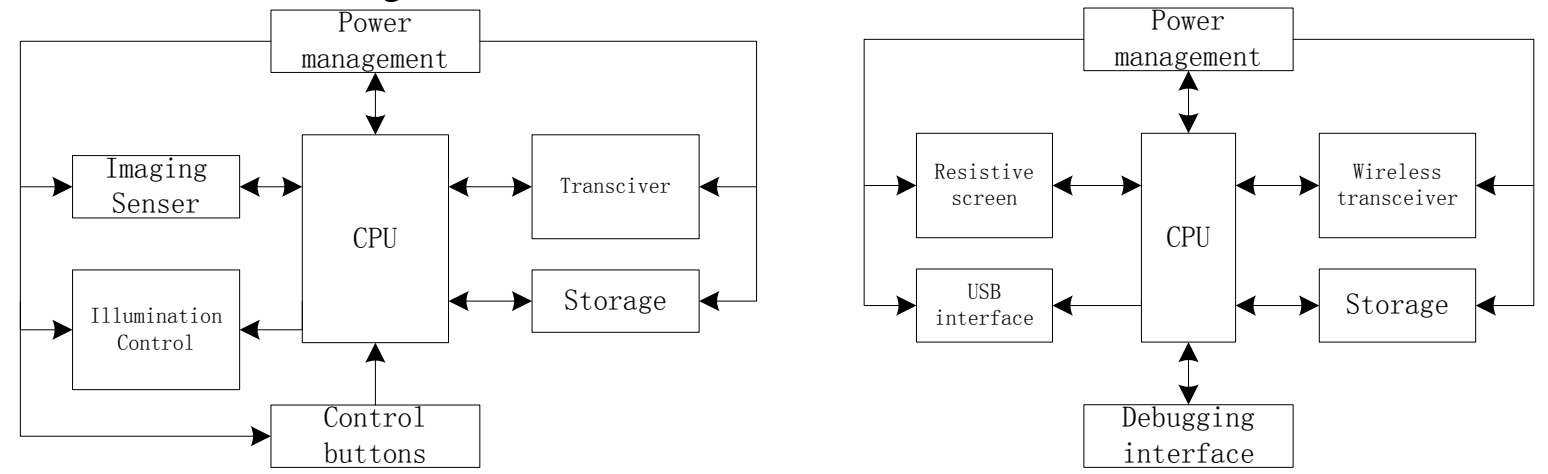

Fig.3. Block diagram of the probe unit. Fig.4. Block diagram of the interactive display unit.

$B$. The function and principle of the interactive display unit.

Interactive display unit has two functions: (1) store and display the images collected by the probe unit and (2) transmit these images to the remote server for being analyzed by doctors and receive their relative feedbacks. Wireless transceiver unit is needed for communicating with the probe, while Ethernet is needed for remote interaction with the server. Because the software for remote interaction is installed in PC, interface between our interactive display unit and PC is only needed to access the internet. USB interface is utilized in our design for connection between interactive 
display unit and PC. For portability, batteries are used for power supply and the resistive screen is used for display. The block diagram of the interactive display unit is shown in Fig.4.

\section{Hardware design and implementation}

A. Hardware circuits design and implementation for the probe unit

Because probe unit is used for collect images inside the oral cavity and is powered by batteries, for hardware design, size and energy consumption should be carefully taken into consideration in addition to the low cost. Synthesizing each kind of situation, a CMOS imaging sensor OV2640 manufactured by Omni Vision Company is chosen as our probe to acquire images of oral cavity. This imaging sensor integrates a data compression engine and can achieve 2 million pixels level, while with only $5.72 * 6.78 \mathrm{~mm}$ size. It supports many output image formats such as RGB, YUV and YCbCR, etc. with only maximum $140 \mathrm{~mW}$ power consumption. The connection with CPU is shown in Fig.5. For wireless transceiver module, we choose nRF24L01 chip manufacture by Nordic Company, which is only $4 * 4 \mathrm{~mm}$ size while achieves $2 \mathrm{Mbps}$ transmission rate. It has simple control interface as well, which is connected to CPU via SPI interface, while has relatively low price. The relevant circuit is shown in Fig. 6.
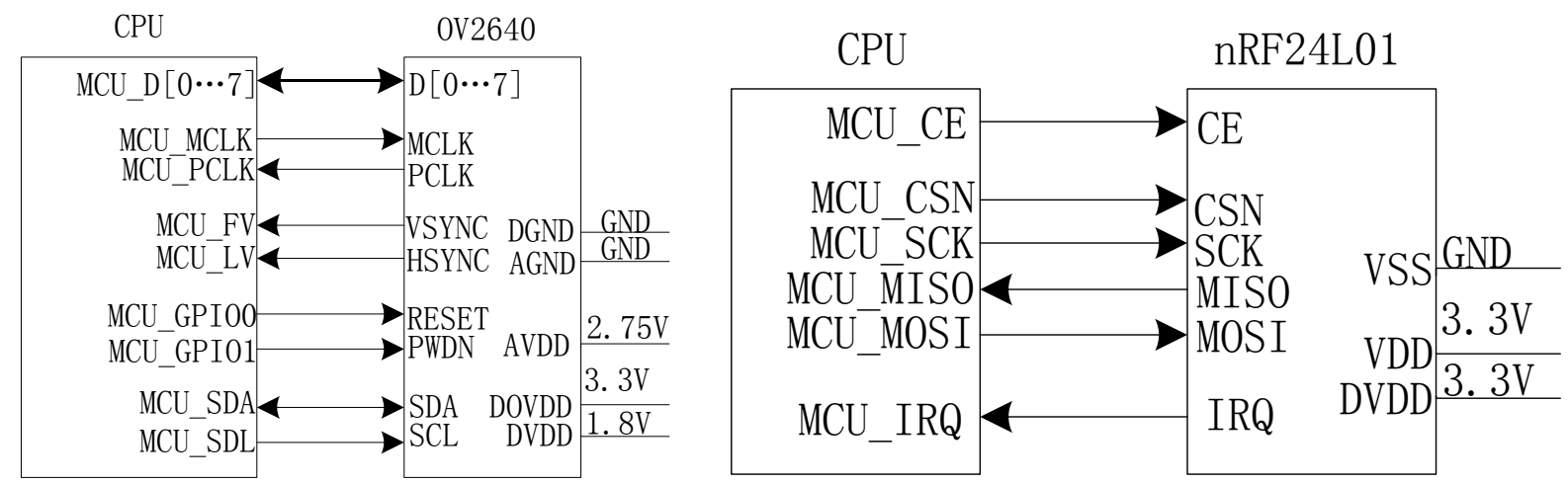

Fig.5. Connection between OV2640 and CPU. Fig.6. Connection between nRF24L01 and CPU.

For power supply, because batteries are used, rechargeable management is designed for avoiding frequently changing batteries. The rechargeable IC for batteries adopts LN2054 and the MF2019 is chosen as our booster circuit to output stable volt at $5 \mathrm{~V}$, which is then stabilized via a Low Drop-Out (LDO) regulator according to the working demand. The relevant circuit is shown in Fig.
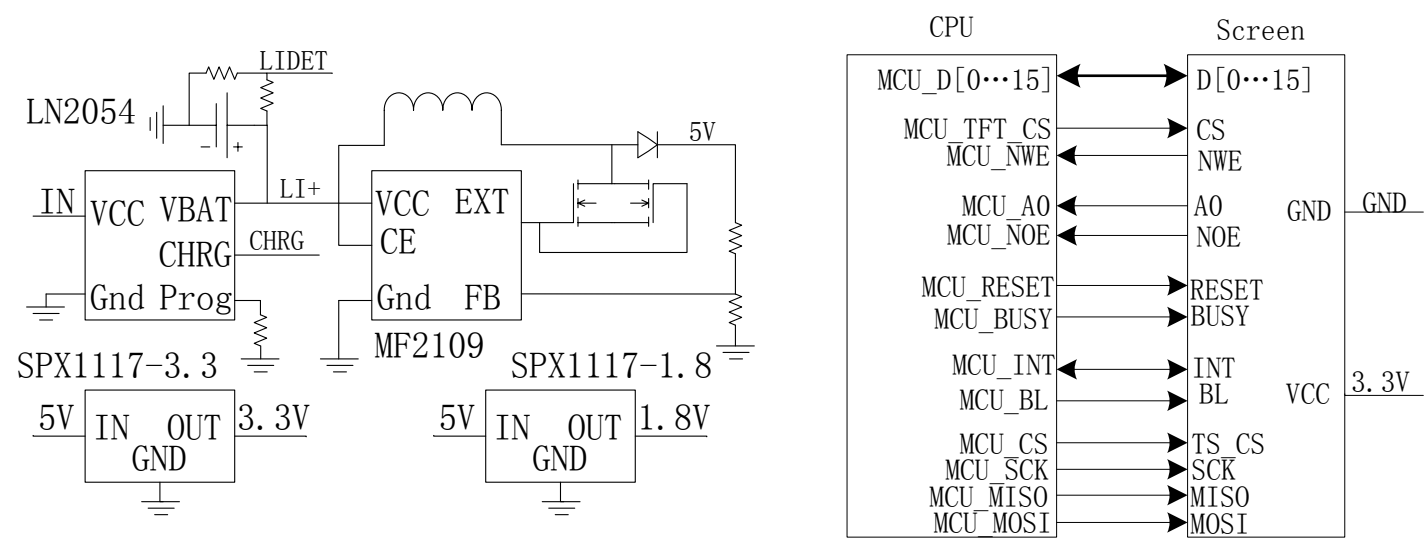

Fig.7. Power supply management circuit.

Fig.8. The circuit of screen interface.

B. Hardware circuits design and implementation for the interactive display unit

Interactive display unit is designed for receive the image data collected by the probe unit and converse the image format for better display on screen. It needs to support USB connection as well. For implementing the requirement above, CPU must have a certain processing speed and size of storage. In our design, STM32F407ZG manufactured by STMicroelectronics Company is chosen as the main processing chip, which embeds a Float Point Unit (FPU) 32ARM CPU with $168 \mathrm{MHz}$ frequency and can reach the performance of 210DIPS/1.25DIPS/MHz (Dhrystone2.1). It includes 
DSP instruction set and has many interfaces as well. The wireless transceiver module uses nRF24L01 which is the same as that in probe. The LCD we use is a 3.2 inch Thin Film Transistor (TFT) LCD with enough resolution of $320 * 240$ pixels. We adopt ILI9230 as the control chip and the interface chip of the touching screen is ADS7846 chip, which is connected to CPU via SPI interface. The relevant circuit and hardware are shown in Fig.8 and Fig.9, respectively.

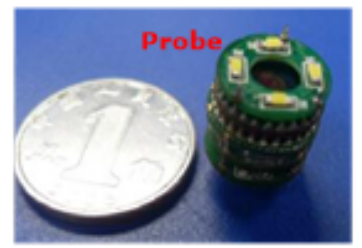

(a)

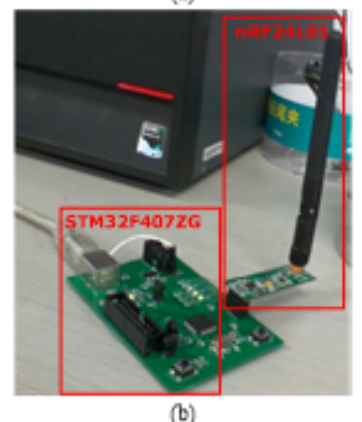

(b)

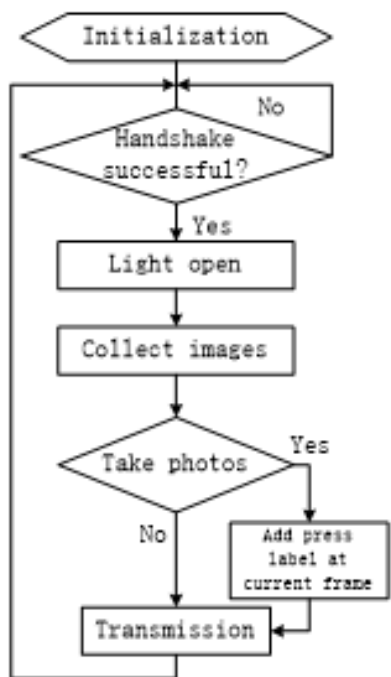

Fig.9. Relevant hardware. Fig.10. Software design of probe. Fig.11. Software design of interactive display unit.

\section{Software design and implementation}

A. Software design and implementation for the probe unit

Software design and implementation for the probe unit include images collection drive software, wireless transceiver drive software, illumination control software and switch control logic. The overall software design is shown in Fig. 10.

The initialization includes configuration of 0V2640 and nRF24L01, illumination and button interrupt. Images are directly sent to the interactive display when there is no button action (press), while a Press Label is added to inform interactive display when press the button to save the images.

B. Software design and implementation for the interactive display unit

Software design and implementation for the interactive display include touching LCD drive software, wireless transceiver drive software, USB drive software, images format conversion software (LCD only supports RGB format, hence, it is necessary to converse the received JEPG images to the RGB format) and etc. The corresponding flow chart is shown in Fig. 11.

The initialization includes the touching LCD initialization, nRF24L01 configuration and USB drive initialization, etc. In our system design, only images rather than videos are supported to transmit under remote mode so as to make sure most of the transmitted images are useful to the doctors.

\section{Results and Analysis}

To test the availability of our system, we combine the probe and interactive display unit together. In our experiment, nRF24L01 chip embedded in the probe transmits the collected images to the interactive display unit. The interactive display unit then sends these received images to the server (PC or other upper machines) for display via USB interface. In order to verify the stability of the wireless communication of our system, we conduct the transmission experiment multiple times, which is to compare the rate of successfully receiving the data frames with and without handshake mechanism. For each scenario, 1000, 1500 and 2000 data frames are collected and transmitted to the interactive display unit. The number of received frames is counted. Table 1 and Table 2 show our experiment results. 
Table 1 Result of transmission stability without handshake mechanism

\begin{tabular}{|c|c|c|c|}
\hline Experiment & NO. of collected frames & NO. of received frames & Success rate \\
\hline 1 & 1000 & 860 & $86.00 \%$ \\
\hline 2 & 1500 & 1235 & $82.33 \%$ \\
\hline 3 & 2000 & 1589 & $79.45 \%$ \\
\hline
\end{tabular}

Table 2 Result of transmission stability with handshake mechanism

\begin{tabular}{|c|c|c|c|}
\hline Experiment & NO. of collected frames & NO. of received frames & Success rate \\
\hline 1 & 1000 & 992 & $99.20 \%$ \\
\hline 2 & 1500 & 1493 & $99.53 \%$ \\
\hline 3 & 2000 & 1987 & $99.35 \%$ \\
\hline
\end{tabular}

From Table 1 and Table 2 we can observe that, the success rate without handshake mechanism is relatively lower, which is unstable for transmission. However, the success rate with handshake mechanism reaches above $99 \%$ for all scenarios, which achieves a very stable transmission. The experiment results show the validity and practicability of our Remote Wireless Intraoral Endoscope System.

\section{Conclusion}

Oral health plays a significant role in people's physical fitness. It is remarkably meaningful to pretend our oral cavity from being harmed. Nevertheless, the incipient symptoms are not obvious and are often ignored by people for the following reasons: (1) several incipient symptoms of oral disease cannot be perceived by people and (2) most of oral inspection equipment is possessed by hospitals or professional outpatient service so that very few of people come to these places for periodical inspection. Motivated by these challenges, a Remote Wireless Intraoral Endoscope System is designed and proposed in this paper for individual, family or community use. People are able to know the status of their oral cavity whenever and wherever possible and can obtain professional feedbacks at home from doctors or specialists. Detailed design of hardware and software are explained in this paper and relative verification experiments of our proposed system are conducted. The experiment results show the validity and practicability of our Remote Wireless Intraoral Endoscope System.

\section{Acknowledgement}

In this paper, the research was sponsored by the Shenzhen Science and Technology Research and Development Funds (Project No.JCYJ20140617143643469).

\section{References}

[1] Chapple I L C, Busby M, Clover H, et al. Periodontal disease and systemic disease[J]. Journal of the Royal Society of Medicine, 2014, 107(3): 94-94.

[2]Halvari A E, Halvari H, Bjørnebekk G, et al. Oral health and dental well - being: testing a self determination theory model[J]. Journal of Applied Social Psychology, 2013, 43(2): 275-292.

[3]Watt R G, Steele J G, Treasure E T, et al. Adult Dental Health Survey 2009: implications of findings for clinical practice and oral health policy[J]. British dental journal, 2013, 214(2): 71-75. 
[4]Miki T, Miyake M, Iwai T, et al. Development of virtual reality training system for oral surgery assisted with the endoscope[J]. International Journal of Oral and Maxillofacial Surgery, 2013, 42(10): 1244-1245.

[5] Neumann H, Fry L C, Nägel A, et al. Wireless capsule endoscopy of the small intestine: a review with future directions[J]. Current opinion in gastroenterology, 2014, 30(5): 463-471.

[6] Peng Y E, Xing C, Hua Z. Design of a Wireless Image Transmission System Based on Microcomputer and nRF24L01[J]. Development \& Innovation of Machinery \& Electrical Products, 2013, 3: 042.

[7]Kuang X H, Wang S M. A Design of Short Range Wireless Communication Module Based on nRF24L01[J]. Applied Mechanics and Materials, 2014, 455: 372-375.

[8] Zhang W, Luo J, Tang X, et al. Fusion Analysis of Electronic Medical Record System and Telemedicine Consultation System[J]. Chinese Medical Record English Edition, 2013, 1(2): 77-80.

[9] Påhlsson H I. The Use of Telemedicine for Performing ERCP[J]. Gastroenterology \&hepatology, 2013, 9(9): 597.

[10]Påhlsson H I, Groth K, Permert J, et al. Telemedicine: an important aid to perform high-quality endoscopic retrograde cholangiopancreatography in low-volume centers[J]. Endoscopy, 2013, 45(5): 357-61.

[11]Shivakumar N S, Sasikala M. Design of vital sign monitor based on wireless sensor networks and telemedicine technology[C]//Green Computing Communication and Electrical Engineering (ICGCCEE), 2014 International Conference on. IEEE, 2014: 1-5. 